\title{
A high resolution spectral atlas of brown dwarfs
}

\author{
A. Reiners ${ }^{1, \star \star}$, D. Homeier ${ }^{1}$, P. H. Hauschildt ${ }^{2}$, and F. Allard ${ }^{3}$ \\ 1 Universität Göttingen, Institut für Astrophysik, Friedrich-Hund-Platz 1, 37077 Göttingen, Germany \\ e-mail: [Ansgar.Reiners; derek] @astro.physik.uni-goettingen.de \\ 2 Hamburger Sternwarte, Gojenbergsweg 112, 21029 Hamburg, Germany \\ 3 Centre de Recherche Astrophysique de Lyon, UMR 5574: CNRS, Université de Lyon, École Normale Supérieure de Lyon, \\ 46 allée d'Italie, 69364 Lyon Cedex 07, France
}

Received 29 May 2007 / Accepted 8 July 2007

\section{ABSTRACT}

\begin{abstract}
We present a UVES/VLT high resolution atlas of three L dwarfs and one T dwarf system, spectral classes at which most of the objects are brown dwarfs. Our atlas covers the optical region from $\mathrm{H} \alpha$ up to the near infrared at $1 \mu \mathrm{m}$. We present spectral details of ultra-cool atmospheres at very high resolution $(R \sim 33000)$ and compare the spectra to model calculations. Our comparison shows that molecular features from $\mathrm{VO}$ and $\mathrm{CaH}$, and atomic features from $\mathrm{Cs}$ and $\mathrm{Rb}$ are reasonably well fit by current models. On the other hand, features due to $\mathrm{TiO}, \mathrm{CrH}$, and water, and atomic $\mathrm{Na}$ and $\mathrm{K}$ reveal large discrepancies between model calculations and our observations.
\end{abstract}

Key words. line: identification - stars: low-mass, brown dwarfs - stars: atmospheres

\section{Introduction}

The analysis of brown dwarfs has seen tremendous progress since their first detection more than ten years ago. Since then, the stellar spectral sequence was augmented by spectral classes $\mathrm{L}$ and $\mathrm{T}$ at its cool end. $\mathrm{L}$ and $\mathrm{T}$ objects must certainly be cooler than those classified as spectral type $\mathrm{M}$, but the reasons for the spectral evolution through the L- and T-classes turned out to be more complex than in the hotter objects (see e.g., Kirkpatrick 2005). The formation of molecules sets in at temperatures typical for spectral classes $\mathrm{K}$ and $\mathrm{M}$, and their opacities begin to cause strong deviations from blackbody spectra. Furthermore, the condensation of dust clouds becomes important at the transition from $\mathrm{M}$ to $\mathrm{L}$ and dominates the appearance of spectral class $\mathrm{L}$ objects. At the $\mathrm{L}$ to $\mathrm{T}$ transition, the atmospheric effects of the dust grains weaken, but this transition is still poorly understood (Burrows et al. 2006).

Objects of spectral class T are always brown dwarfs according to standard evolutionary models (Chabrier \& Baraffe 2000). Depending on age, however, objects of spectral classes $\mathrm{M}$ or $\mathrm{L}$ may be either brown dwarfs or hydrogen-burning stars. Young brown dwarfs can be found in the same temperature range as old low-mass stars, hence it is not possible to determine brown dwarf status from the spectral type (taken as proxy for effective temperature) alone (Basri 2000; Chabrier et al. 2005).

As brown dwarfs are defined by mass, there is no obvious atmospheric feature that determines brown dwarf status by just looking at an object's spectrum. While the Li-test (e.g., Basri 2000) is a useful tool in young objects, it is not suitable for the majority of old (field) objects. Age can as well be determined in members of stellar clusters, although by today this is only

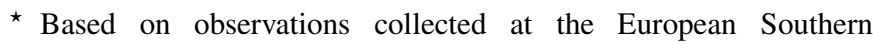
Observatory, Paranal, Chile, 077.C-0449.

$\star \star$ Emmy Noether Fellow.
}

useful for young brown dwarfs because they are bright enough to be observed at the large distance of the clusters. A direct determination of brown dwarf status is possible for members of binary systems through the measurement of their mass, but that is not possible for most field objects. The obvious way to determine the mass of an ultra-cool object is through its gravity (e.g., Mohanty et al. 2004). The mass can directly be calculated once gravity and radius are known, and the latter can be obtained from the luminosity, a parameter that is relatively well constrained. Burgasser et al. (2006) have shown a method to determine the gravity in $\mathrm{T}$ dwarfs, and they calculate masses for $13 \mathrm{~T}$ dwarfs. These authors determine gravity from color ratios in low resolution spectra that they calibrated from model calculations. They claim rather low uncertainties for their results, but one has to keep in mind that this method is strongly dependent on the match between calibration models and observed spectra (see also discussion of uncertainties in Burgasser 2007). The models of ultra-cool atmospheres, however, are still evolving, and "the extraction of physical quantities such as $T_{\text {eff }}$, gravity, and metallicity from the growing library of well-calibrated spectra, although possible in a crude sense, remains imprecise" (Burrows et al. 2006).

The rapid progress in the investigation of (ultra-)cool atmospheres is due to the advent of $10 \mathrm{~m}$ class telescopes that allow spectroscopy in these faint targets. The redward shift of the spectral energy distribution (SED) in cool stars moves the peak of the SED out of the range of "classical" optical spectrographs into the IR domain. Furthermore, the drop in temperature is connected to a loss in luminosity with $T^{4}$, and both effects together render it very difficult to obtain high quality optical spectroscopic data for the coolest stars and brown dwarfs.

Kirkpatrick et al. (1991) have shown low resolution red and near-IR spectra in mid- $\mathrm{K}$ and $\mathrm{M}$ dwarfs, and they have also identified a great many atomic lines and molecular bands. Optical 
Table 1. Objects observed for this atlas and temperatures adopted for the models.

\begin{tabular}{ccccc}
\hline \hline Object & SpType $^{a}$ & $\begin{array}{c}J^{b} \\
{[\mathrm{mag}]}\end{array}$ & $\begin{array}{c}\text { exp. time } \\
{[\mathrm{s}]}\end{array}$ & $\begin{array}{c}T_{\text {model }}{ }^{c} \\
{[\mathrm{~K}]}\end{array}$ \\
\hline 2MASS 1731+2721 & L0 & 12.09 & 2400 & 2200 \\
2MASS 1155-3727 & L2 & 12.81 & 5200 & 2000 \\
2MASS 1507-1627 & L5 & 12.83 & 5200 & 1700 \\
2MASS 2204-5646 & T1+T6 & 11.91 & 3000 & 1280 \\
$(\epsilon$ Indi B $)$ & & & & \\
HD 114772 & B9V & $V=5.89$ & 25 & \\
\hline
\end{tabular}

${ }^{a}$ Optical spectral type from DwarfArchives.org; ${ }^{b} 2$ MASS system; ${ }^{c}$ See Sect. 3.

low resolution spectra of $\mathrm{T}$ dwarfs together with identifications of the main absorption features were provided by Burgasser et al. (2003). For the IR wavelength range, McLean et al. (2003) and Cushing et al. (2005) published compilations of low resolution spectra in M, L and T dwarfs, and McLean et al. (2007) also showed high resolution IR spectra for these spectral classes.

High resolution spectra of ultra-cool stars and brown dwarfs are difficult to obtain. This is a pity because it is the pressure dependent wings of the saturated atomic absorption lines (predominantly of the alkali elements) that are most sensitive to gravity. While the strong alkali lines depend on effects like temperature, metallicity, rotation, etc. as well, these parameters can be determined from molecular bands, which can resolve the degeneracy between gravity and other parameters. In high resolution spectra, several features are available to constrain atmospheric parameters simultaneously. Thus, the determination of gravity from the pressure broadened wings can be expected to be much more accurate than comparing color ratios from low resolution spectra. High resolution optical spectra of M dwarfs were shown by Tinney \& Reid (1998), but no high resolution data showing the full optical spectrum in L dwarfs or cooler were made available. Some individual features in high resolution $\mathrm{L}$ dwarf spectra were investigated by Basri et al. (2000); Schweitzer et al. (2001, 2002).

In this paper, we present an atlas of high resolution, high signal to noise spectra in the red and near-IR spectral range for three $\mathrm{L}$ and an early $\mathrm{T}$ dwarf $\operatorname{system}\left(2200 \gtrsim T_{\text {eff }} \gtrsim 1200 \mathrm{~K}\right)$. We also compare a set of the most recent model calculations to the data. For the full wavelength region, we show the spectral features of ultra-cool $\mathrm{L}$ and early $\mathrm{T}$ dwarfs at high resolution, and we make a first attempt to identify the shortcomings and successes of our current model calculations.

\section{Data}

\subsection{Objects and format of the atlas}

We present a high resolution spectral atlas of four very low mass objects of spectral types between L0 and T1. Name, spectral type, $J$-magnitude, and exposure times of the objects are given in Table 1. The signal to noise ratio (SNR) of the spectra varies over the wavelength region according to the object's spectral energy distribution and detector efficiency, it is well over 20 in most of the region in the $\mathrm{L}$ dwarfs, so that the dense molecular absorption features are well discernible from the noise.

In Fig. 1, we show the full spectra of all four targets. In the top panel, we also provide the spectrum of a rapidly rotating B9V star (also listed in Table 1) that we observed with the same setup to identify telluric lines due to absorption of Earth's atmosphere. In Figs. 2 we provide the detailed spectral atlas with identifications of the most prominent absorption features. In all plots, synthetic spectra chosen to match the object's effective temperature are overplotted. We discuss the models in Sect. 3.

\subsection{Observations}

Data were obtained with the UVES spectrograph at ESO/VLT in service mode during April and May, 2006. UVES was operated in dichroic mode using both the blue and the red arm. Because of the red colour of L dwarf spectra, the blue instrument arm contains very little flux if any, but it covers several hydrogen lines that are interesting during flares. For this spectral atlas, we only consider the red arm that was configured in a non-standard setting centered at $830 \mathrm{~nm}$. This setting covers the wavelength region 6400-10200 $\AA$, it contains $\mathrm{H} \alpha$ and the FeH Wing-Ford band that is very useful for spectral analysis in late-type dwarfs (Reiners \& Basri 2006a). Between the two CCDs of the red arm, the spectra have a gap from $8200 \AA$ to $8370 \AA$. The Na doublet at $8190 \AA$ lies just blueward of the gap. The spectra were taken at a slit width of 1 '.2 yielding a resolving power of $R \sim 33000$. Data were reduced using the MIDAS-based ESO-pipeline for UVES data. Bias-subtraction, division by a flatfield-lamp spectrum, and wavelength calibration follow standard routines. We did not remove telluric lines for our spectral atlas, but we show a spectrum of a telluric standard star for comparison.

\section{Models}

Theoretical models have been calculated using the generalpurpose stellar atmosphere code PHOENIX version 15.2. Details of the numerical methods are given in Hauschildt \& Baron (1999). In this work we use a setup of the microphysics that gives the currently best fits to observed spectra of M, L, and T dwarfs for the low $T_{\text {eff }}$ regime and that also updates the microphysics used in the GAIA model grid (Kučinskas et al. 2005, 2006). The water lines are taken from the calculations of Barber et al. (2006); this list gives the best overall fit to the water bands over a wide temperature range. TiO lines are taken from Schwenke (1998) for similar reasons. The treatment of opacities and the equation of state (EOS) is similar to the one described in detail in Allard et al. (2001), with extensions and modifications described below (HITRAN database ${ }^{1}$; Plez, Bernath, priv. comm.).

One of the most important recent improvements of cool stellar atmosphere models is the availability of new atomic line profile data based on accurate inter-atomic potentials. The calculations presented here include detailed and depth dependent line profiles for each of the D1 and D2 transitions of the Alkali resonance doublets ( $\mathrm{Li}, \mathrm{Na}, \mathrm{K}, \mathrm{Rb}, \mathrm{Cs}$ ) for perturbation by both $\mathrm{H}_{2}$ and He, as described in Allard N. F. et al. (2003); Allard et al. (2005); Allard \& Spiegelman (2006); Allard et al. (2007a,b). We also include damping of the $\mathrm{Na}$ and $\mathrm{K}$ lines by HI using the broadening widths of Andretta et al. (1991); Leininger et al. (2000) and Barklem \& O'Mara (2001), though for L dwarf temperatures atomic hydrogen only accounts for less than $10 \%$ of the total broadening (Johnas et al. 2007).

These profiles give a much improved representation of the details of the line shapes in the optical spectra of $\mathrm{M}, \mathrm{L}$ and $\mathrm{T}$ dwarfs, and become especially important in situations where line blanketing and broadening are crucial for the model

\footnotetext{
${ }^{1}$ http://www.cfa.harvard.edu/HITRAN/
} 


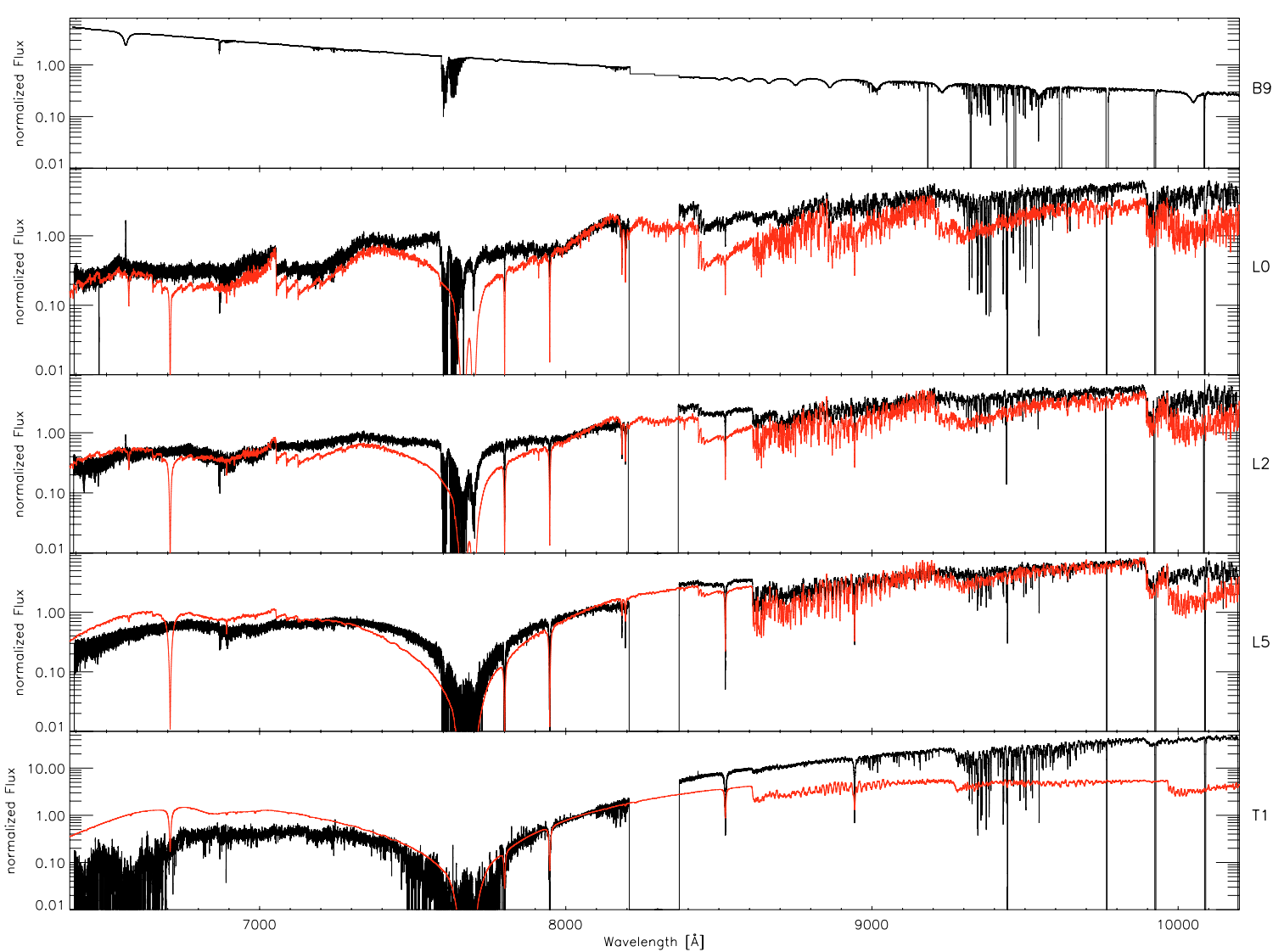

Fig. 1. Full spectra (black) and models (red) of 2M1731 (L0), 2M1155 (L2), 2M1507 (L5), and 2M2204 (T1). For comparison, a telluric reference star (spectral type B9V) is shown.

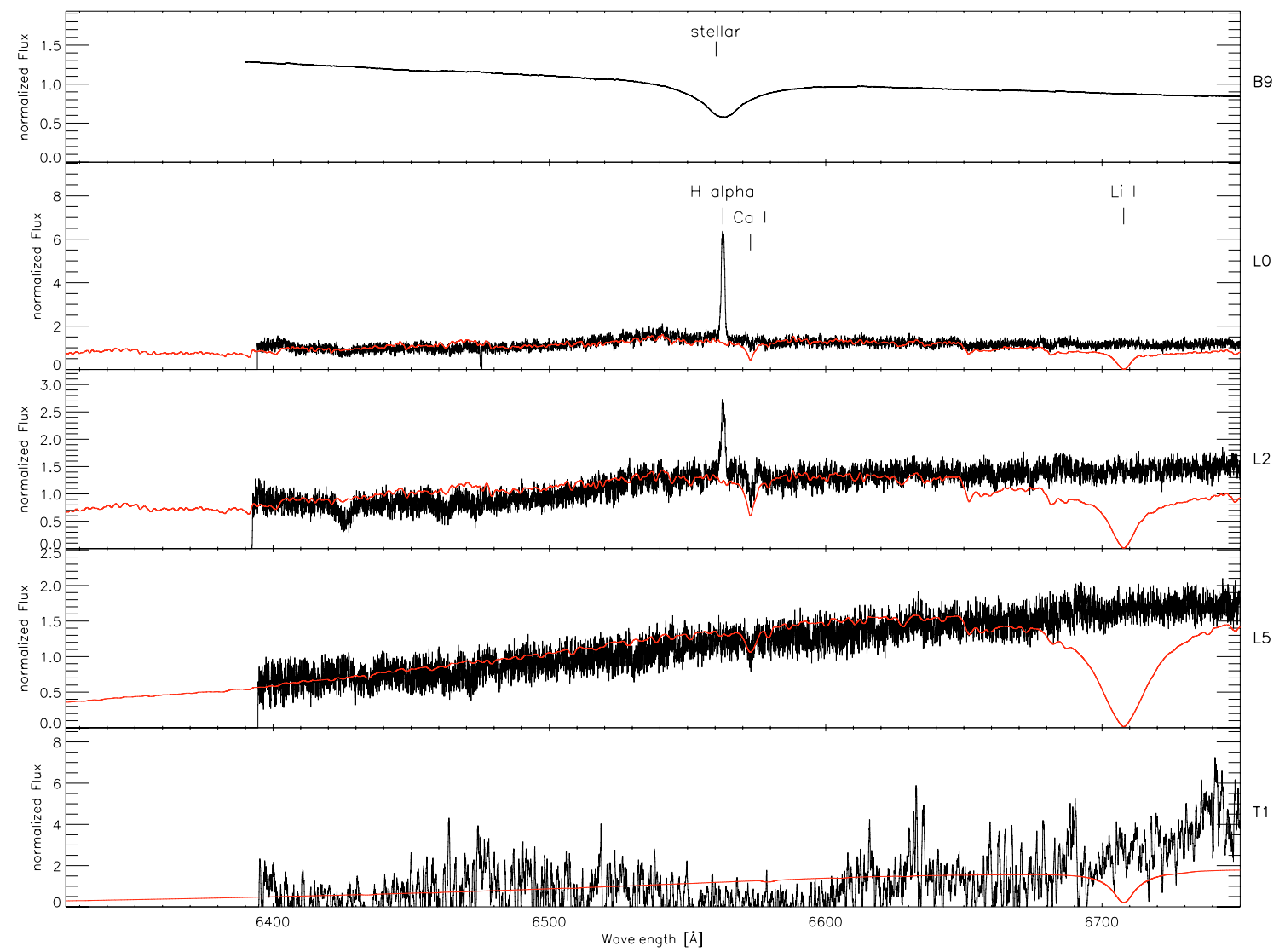

Fig. 2. Spectral atlas (black) and models (red) of 2M1731 (L0), 2M1155 (L2), 2M1507 (L5), and 2M2204 (T1). For comparison, a telluric reference star (spectral type B9V) is shown. 


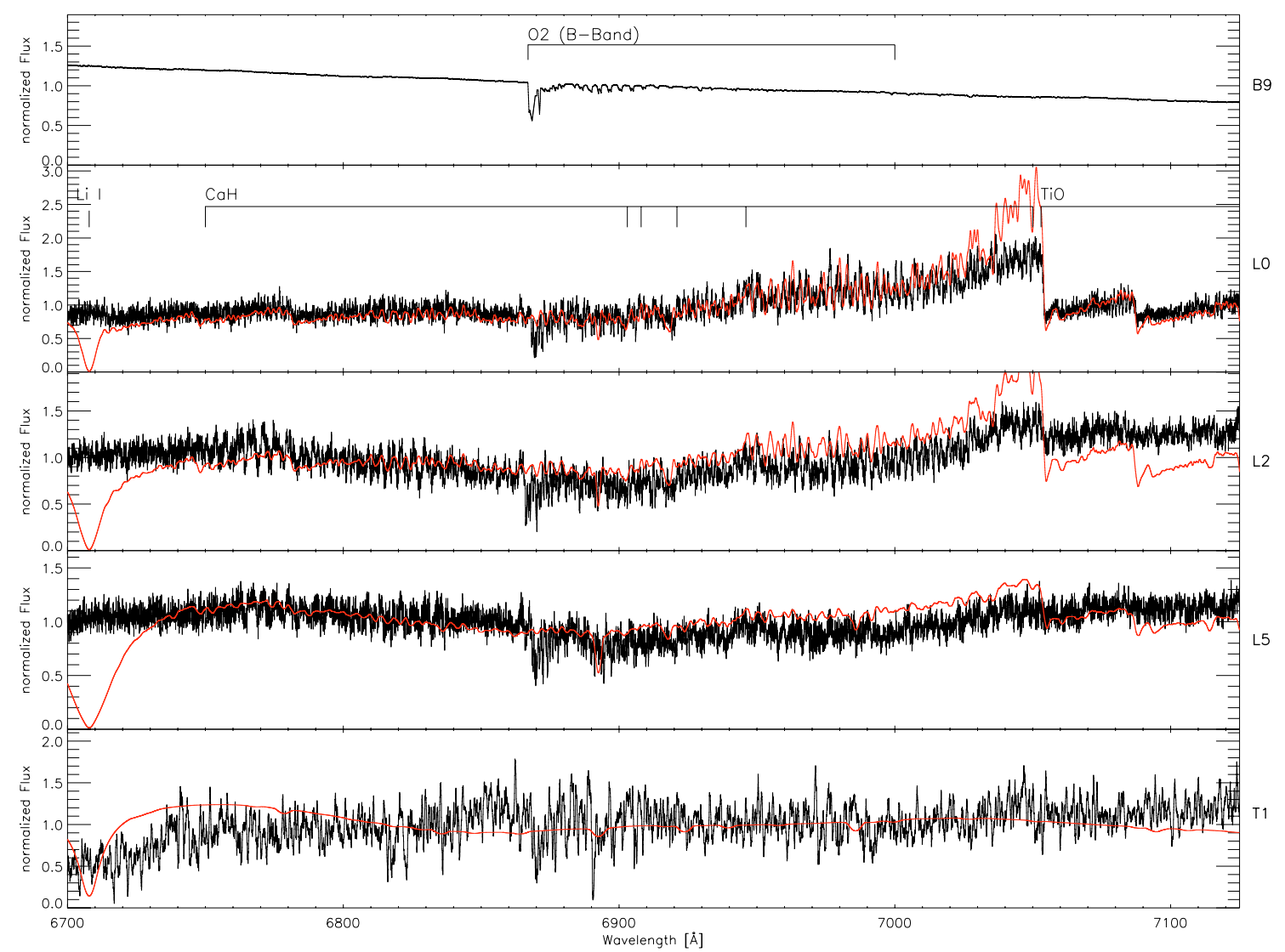

Fig. 2. continued.

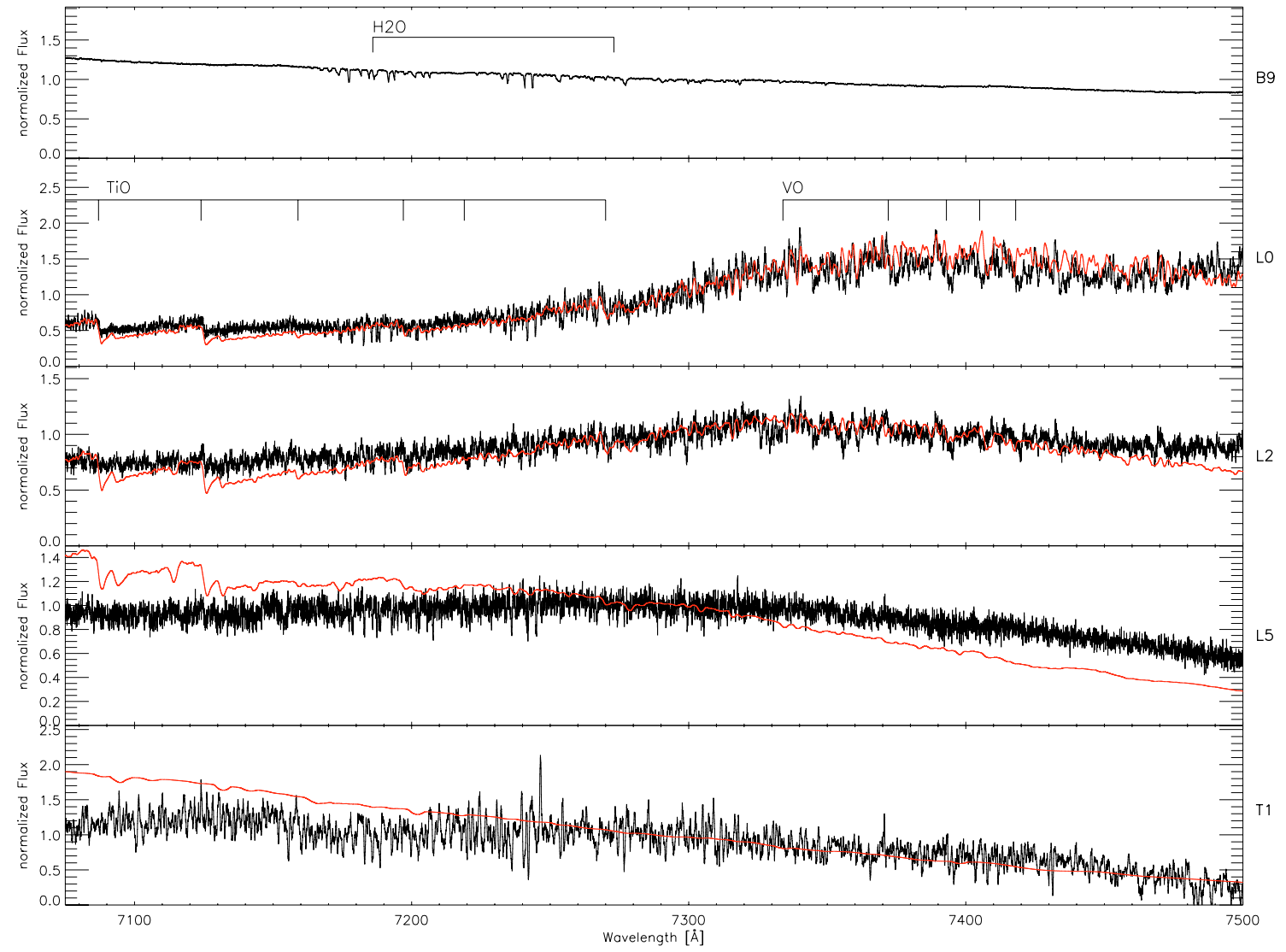

Fig. 2. continued. 


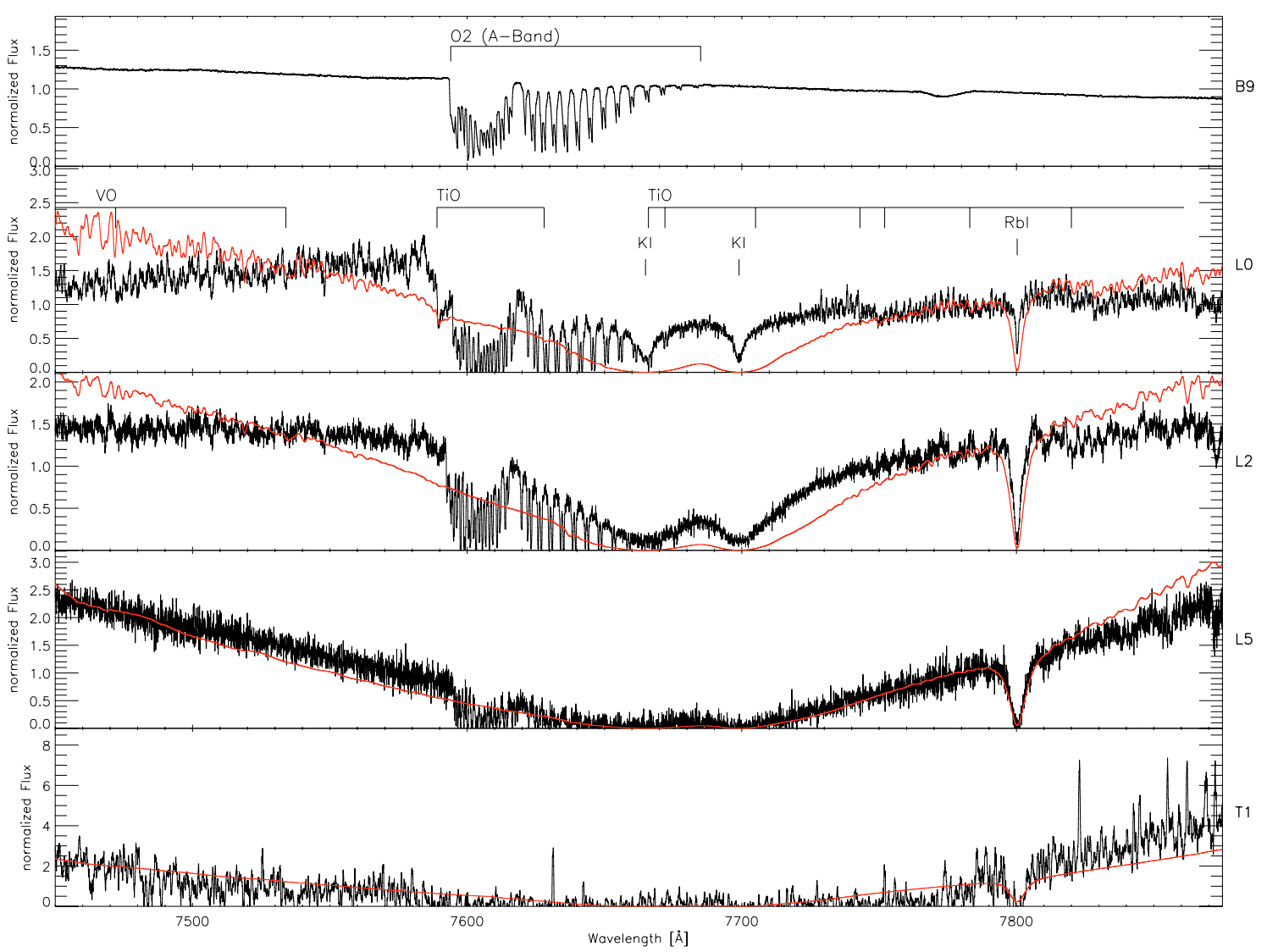

Fig. 2. continued.

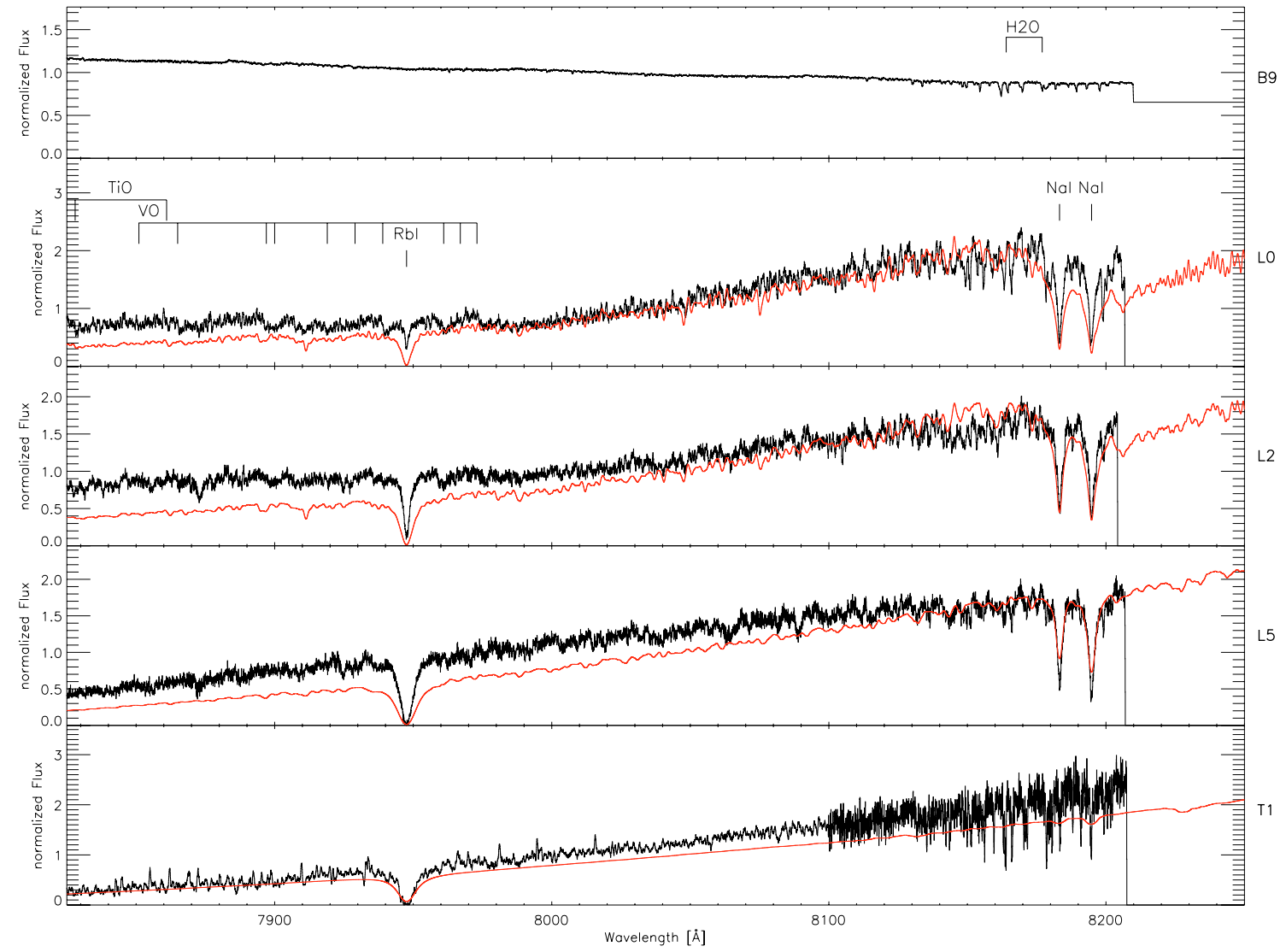

Fig. 2. continued. 


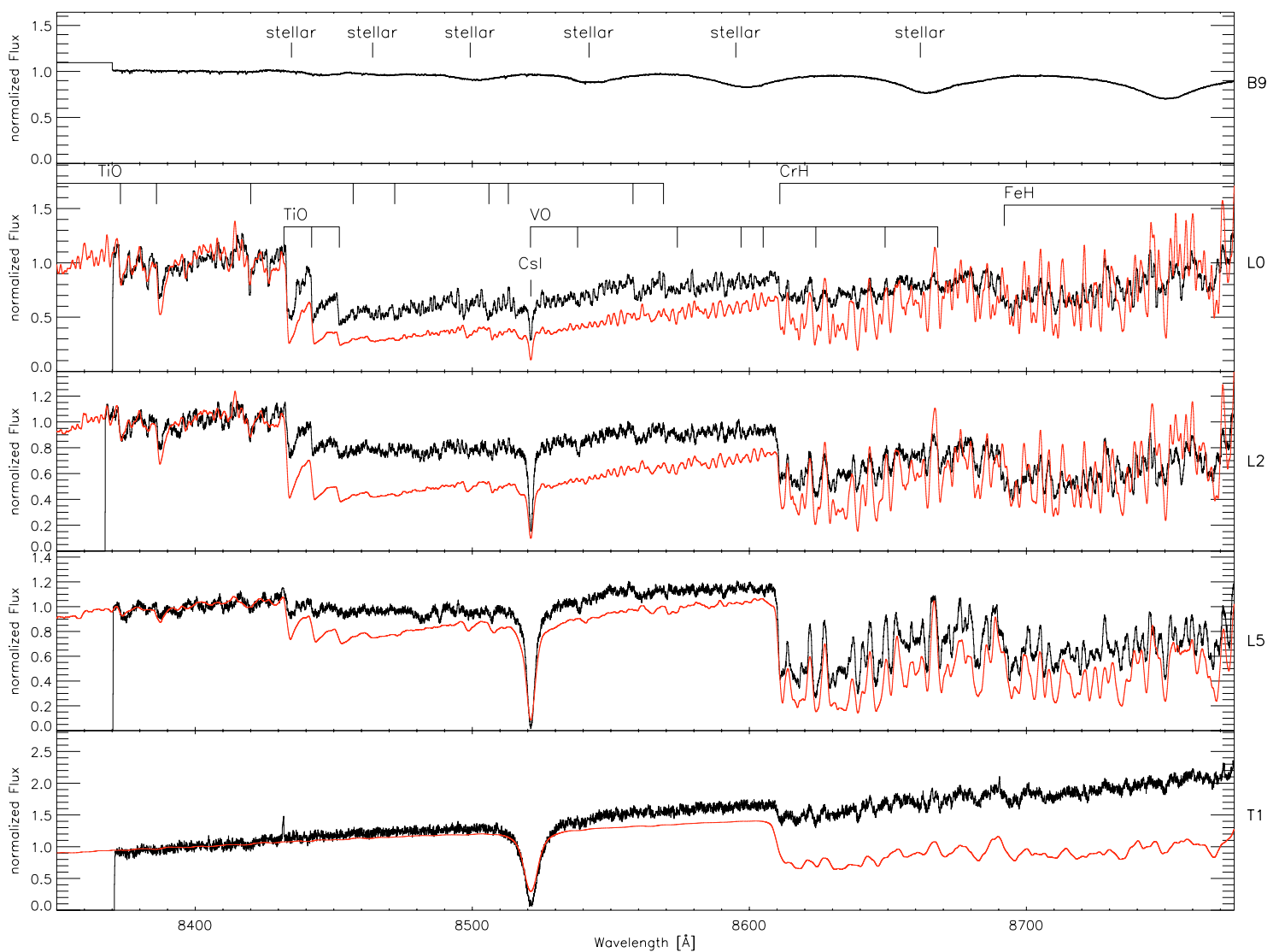

Fig. 2. continued.

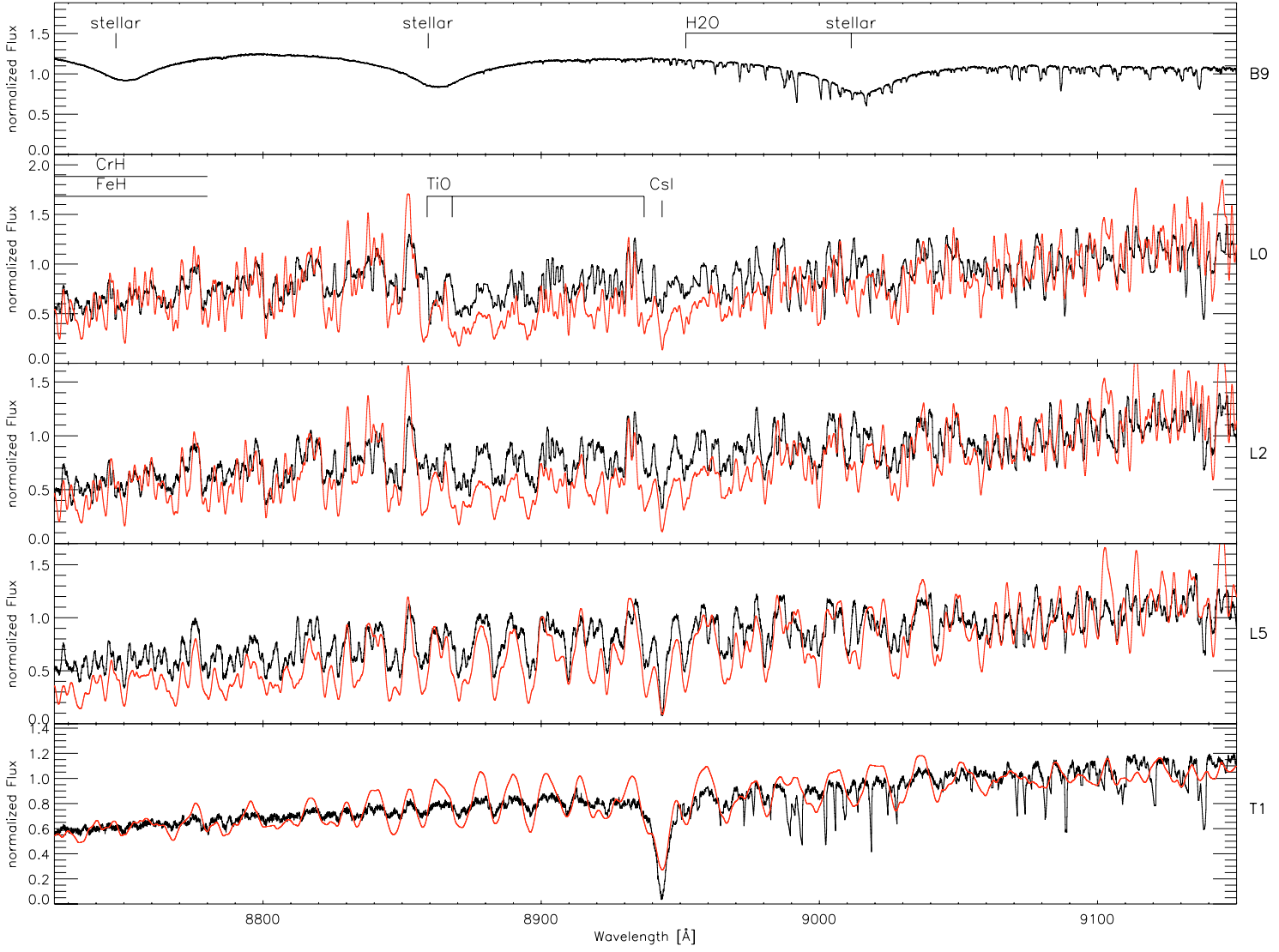

Fig. 2. continued. 


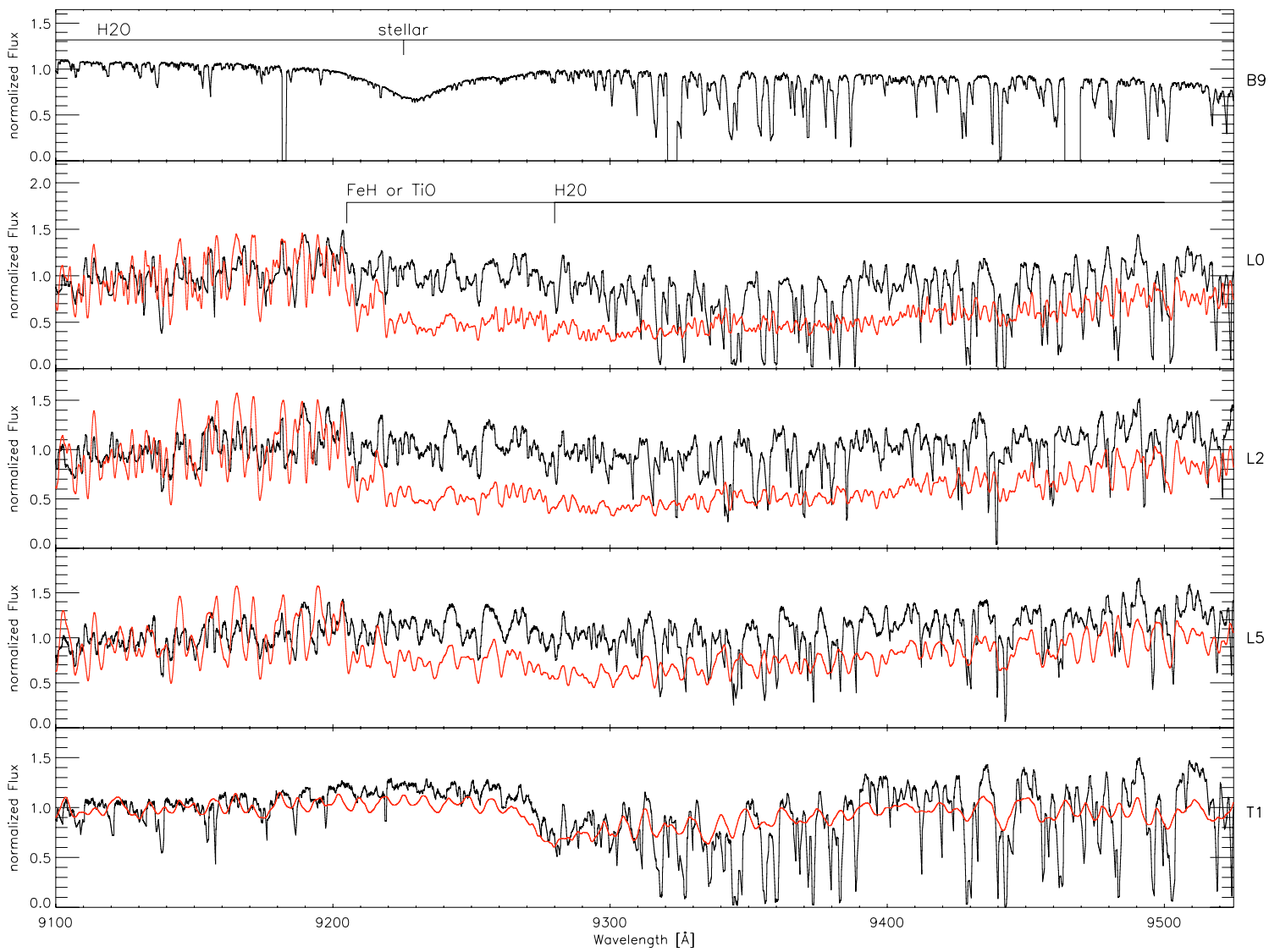

Fig. 2. continued.

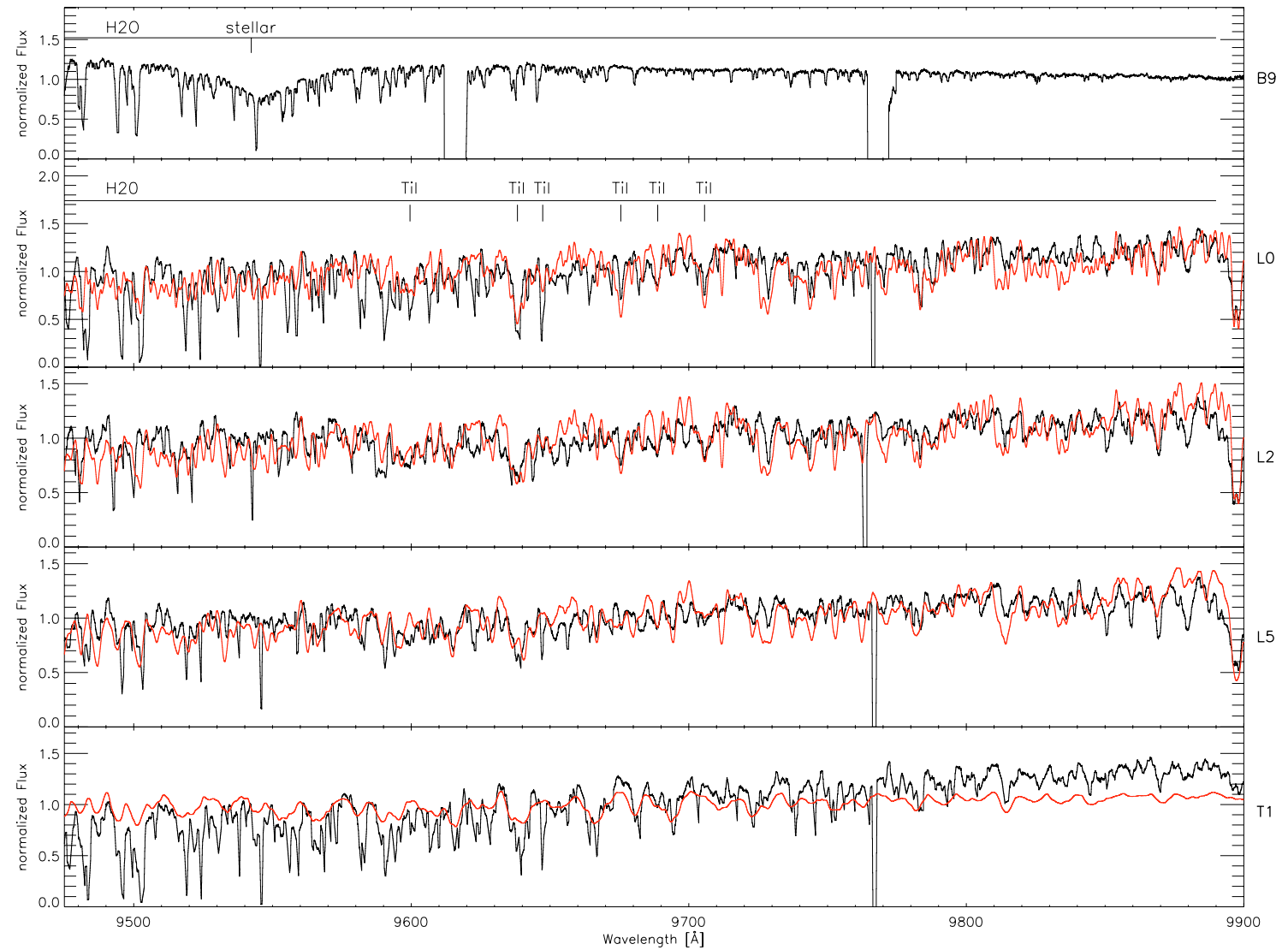

Fig. 2. continued. 


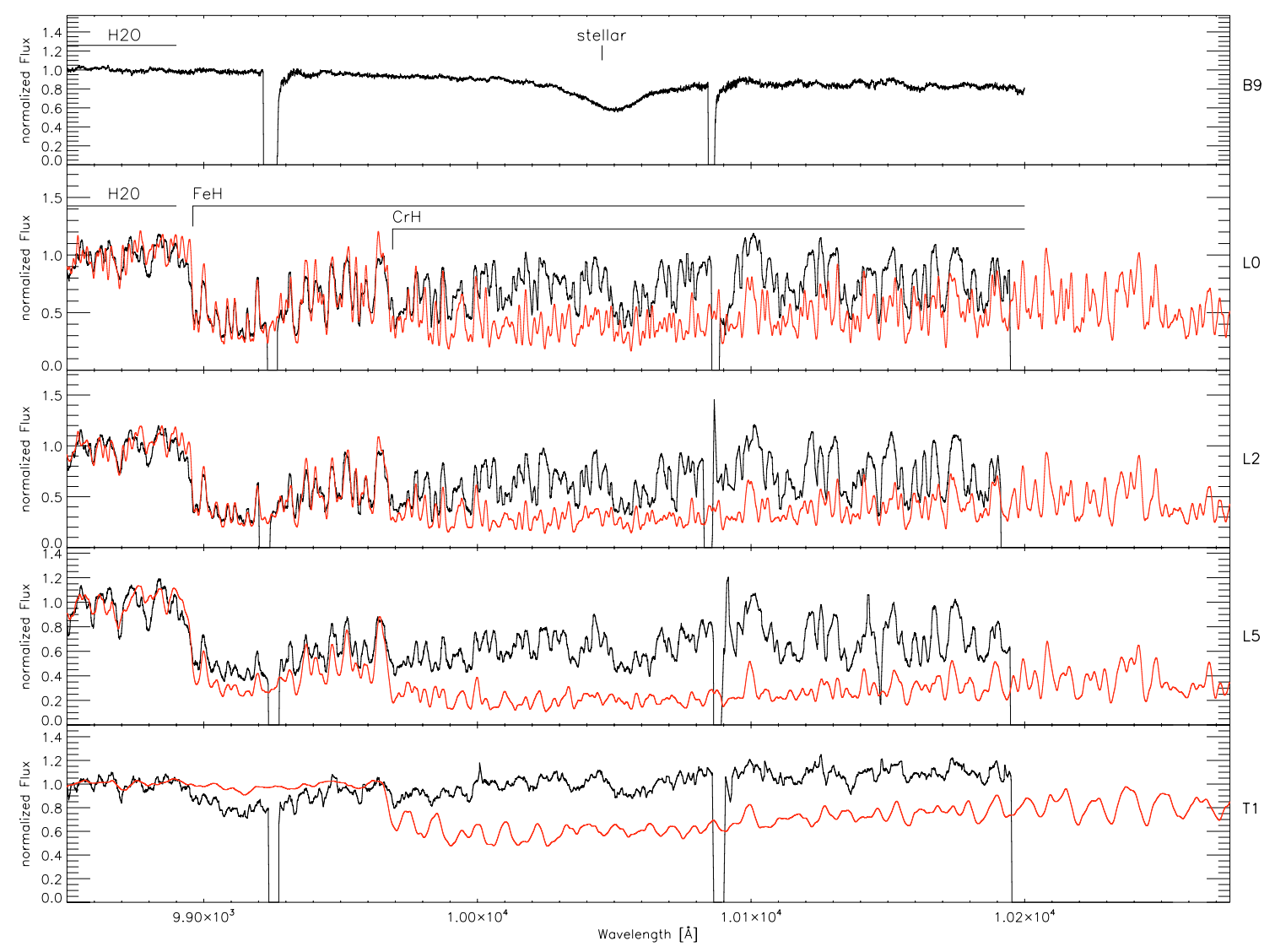

Fig. 2. continued.

structure calculations and for the computation of the synthetic spectra, i.e. most notably in $\mathrm{T}$ dwarfs, where dust as a source of continuum opacity becomes less important.

The EOS is an updated version of the one used in Allard et al. (2001), we include about 1000 species (atoms, ions and molecules). The EOS calculations themselves follow the method discussed in Allard \& Hauschildt (1995). For effective temperatures $T_{\text {eff }}<2500 \mathrm{~K}$, the condensation of dust particles has to be considered in the EOS. In our models we allow for the formation (and dissolution) of a variety of grain species. For details of the EOS and the opacity treatment of condensates see Allard et al. (2001).

It has long been evident that the "Dusty" limit described in Allard et al. (2001), which assumes the presence of condensates everywhere where they are thermodynamically stable, becomes increasingly inaccurate in the case of late $\mathrm{L}$ and especially $\mathrm{T}$ dwarfs. A more realistic treatment needs to include the gravitational settling, sedimentation or rainout, of condensates, which results in a cloud layer retreating to larger optical depths as $T_{\text {eff }}$ decreases (Allard F. et al. 2003). While these processes become very noticeable at $T_{\text {eff }}$ below $2000 \mathrm{~K}$, the settling of dust in high altitudes and the associated depletion of condensable elements can affect the spectra already much earlier for features that form at very low optical depths, such as strong molecular bands and the cores of the $\mathrm{Na}$ I and $\mathrm{K}$ I resonance lines. Lodders $\&$ Fegley (2006) have shown that the sequential removal of refractory species in order of decreasing condensation temperature - the "rainout" scenario - leads to different chemical composition from the full chemical equilibrium (CE) treatment, because in the former case high temperature condensates would remove their constituent elements from the gas phase in deep layers of the atmosphere, thus cutting off all higher layers from the supply of these elements. The formation of lower condensation temperature species, that would form in a fully mixed gas in CE, could thus be inhibited. One such case potentially relevant for brown dwarf atmospheres is the formation of anorthite $\left(\mathrm{CaAl}_{2} \mathrm{Si}_{2} \mathrm{O}_{8}\right)$, which would allow alkali metals to condense into feldspars (e. g. albite, $\mathrm{NaAlSi}_{3} \mathrm{O}_{8}$, orthoclase, $\mathrm{KAlSi}_{3} \mathrm{O}_{8}$ ) at $T<1200 \mathrm{~K}$. However, other calcium silicates can form at much hotter temperatures, as soon as at the $1700 \mathrm{~K}$ level. Thus, in a fully rained out atmosphere, it will remove all $\mathrm{Ca}$ from the gas phase correspondingly deeper in the atmosphere, and from all layers above that level. Anorthite would thus not get a chance to form. Therefore, in such a chemistry the first alkali-bearing condensates to appear are the alkali halides at $\sim 1000 \mathrm{~K}$. The alkali metals could then only be depleted at higher and cooler layers than in the CE case, where feldspars could form around $\sim 1200 \mathrm{~K}$.

In a real (sub-) stellar atmosphere neither of these limiting cases may be realised, since the fractionation of condensates by gravitational settling and turbulent mixing compete with each other and both effects have to be accounted for. We have therefore consistently used the latest version of the Settling models, which model the vertical distribution of condensates by comparing the dynamical timecales of dust condensation, growth and sedimentation with vertical mixing by convective overshoot. The overall shape of the velocity field in the overshoot region, which is responsible for supporting cloud layers in the radiative zone, is based on a limited set of 3D-hydrodynamical simulations (Ludwig et al. 2006). Results of these simulations are generalised to different atmospheric temperatures and gravities using the velocities predicted by mixing length theory within the convectively unstable region, and extrapolating from there with an exponential relation based on the hydrodynamical 
models. The main free input parameter in our models controls this parametrisation of the overshoot velocity and is chosen to match the observed changes of dust signatures along the $\mathrm{L}$ and $\mathrm{T}$ spectral sequences.

Atmosphere structures for the models were computed solving the radiative transfer on a medium-resolution wavelength grid of 1-2 A spacing for the optical to near-IR region. From the converged structures high-resolution spectra with a sampling of $0.05 \AA$ were produced in one or two additional iterations, checking for temperature corrections and flux error to ensure that the final spectral energy output was consistent with the calculated structure. We did not fit $T_{\text {eff }}$ and $\log g$ to obtain the best match to the observed spectra, since modelling of high-resolution spectra is known to produce misleading results for these fundamental parameters (Schweitzer et al. 2001; Reiners 2005). Instead we picked the effective temperature according to the spectral class $-T_{\text {eff }}$ relation of Vrba et al. (2004); Golimowski et al. (2004), which is based on absolute luminosity and therefore is much less subject to modelling uncertainties. The L dwarfs in this study, as isolated field brown dwarfs, are likely to be fairly old (at least several $10^{8}$ years) and massive, an assumption confirmed by the absence of lithium in their spectra (cf. 4.2.1). Evolutionary models (Chabrier et al. 2000; Baraffe et al. 2002) predict gravities in the range $\log g=5.2 \ldots 5.4$ for such objects. Therefore a single $\log g=5.25$ has been used for all models, except for $\epsilon$ Indi B, where $T_{\text {eff }}$ and $\log g$ are much better constrained by the photometry, parallax and age estimate of McCaughrean et al. (2004).

\section{Spectral features and comparison to models}

In this section, we discuss the key $\mathrm{L}$ and $\mathrm{T}$ dwarf features shown in our spectral atlas in Figs. 2. We compare the data to the modeled spectra and try to identify the improvements necessary to obtain a better fit. We discuss the molecular features in Sect. 4.1 before we turn to the atomic lines in Sect. 4.2.

\subsection{Molecular bands}

\subsubsection{Metal oxides}

$\mathrm{TiO}$ and VO are the most important opacity sources in M stars. They remain strong in the L dwarfs, but get weaker towards cooler temperature due to condensation into dust species like perovskite $\left(\mathrm{CaTiO}_{3}\right)$, solid titanium oxides and vanadium oxides, respectively (see e. g. Lodders 2002; Ferguson et al. 2005). Our spectra cover the TiO bandheads at $7053 \AA$, $7589 \AA$, $7666 \AA$, $8432 \AA$, and $8859 \AA$. All bands are recovered by the models, but the $\mathrm{TiO}$ bands are too strong in the theoretical spectra particularly in the L2 and L5 dwarfs. This hints at remaining shortcomings in our treatment of dust settling (see Sect. 3), which evidently predicts too much $\mathrm{TiO}$ to be left in the atmosphere at temperatures between 1500 and $2000 \mathrm{~K}$. VO bands are visible at $7334 \AA, 7851 \AA$, and $8521 \AA$. In general, VO bands match the data quite well, but a detailed comparison is hampered by the mismatch of the $\mathrm{TiO}$ bands, which often affects the same spectral regions.

Another uncertainty that may be responsible for parts of the mismatch between our data and the model spectra are the (TiO) absorption bands' oscillator strengths (Allard et al. 2000; Reiners 2005). Laboratory experiments do not yet provide values at the accuracy required in order to match the data, and further improvement on the molecular data is needed.

\subsubsection{Metal hydrides}

The three metal hydride species $\mathrm{CaH}, \mathrm{FeH}$, and $\mathrm{CrH}$ are important in the optical spectra of L dwarfs. $\mathrm{CaH}$ at $6750 \AA$ is visible in the L dwarfs and is accurately reproduced by the models. The excellent match between the LO model and the spectrum shows that the high frequency patterns visible at this spectral resolution (around $7000 \AA$ ) is the structure of the absorption band and not noise. These patterns disappear around L5, confirming that $\mathrm{CaH}$ is depleted at later spectral types and is superseded by the $\mathrm{K} \mathrm{I}$ satellite feature (see Sect. 4.2.1).

The general structure of the $\mathrm{CrH}$ band at $8611 \AA$ also matches quite well the structure of the data in all three L dwarfs and also in the T dwarf. Unfortunately, this band is embedded in the strong TiO and VO bands, which the models overpredict, as well as FeH. The calculated strength of the $\mathrm{CrH}$ hence is difficult to judge in the L dwarfs (see below).

$\mathrm{FeH}$ is observed close to the $\mathrm{CrH}$ band at $8692 \AA$ and extends to the red all the way to $>1 \mu \mathrm{m}$, but is increasingly superposed by $\mathrm{CrH}$, and between $8861-9334 \AA$ by TiO. The models compare to the data as good as for $\mathrm{CrH}$. The prominent Wing-Ford band of $\mathrm{FeH}$ is visible at $9896 \AA$. Structure and intensity of this band are well fit by the model at the first few Ångstrom, in early L dwarfs. However, this band is overestimated at very low temperature as well. At $9969 \AA$, another $\mathrm{CrH}$ band should be visible. It is contained in the model spectra dominating the absorption redwards of $9969 \AA$ in the L5 and T1 spectra. However, Reiners \& Basri (2006a) show that all absorption features at that wavelength region can be explained by $\mathrm{FeH}$ in $\mathrm{M}$-dwarfs, they see no evidence for $\mathrm{CrH}$ absorption. In the models shown here, $\mathrm{CrH}$ produces a strong step in the model spectra for the L5 and T1 objects. Such a feature at $9969 \AA$ is not observed in our data, or at least at much less strength, supporting the argument that the absorption around $1 \mu \mathrm{m}$ in early L dwarfs is entirely due to $\mathrm{FeH}$, and that $\mathrm{CrH}$ is much weaker than predicted.

A similar case can be made for the $8692 \AA$ band. While for the earlier Ls the agreement with the observed band seems reasonably good, in the T dwarf system it is much too strong in the model. A more detailed investigation of the molecular line formation in the models reveals that at higher $T_{\text {eff }}$ both $\mathrm{FeH}$ and $\mathrm{CrH}$ contribute in similar shares to the absorption all the way to the blue end, with $\mathrm{FeH}$ being mostly responsible for the stronger lines and the structure of the band. In the $1280 \mathrm{~K}$ model however, settling effects have already removed significant quantities of the hydrides from the line forming region. $\mathrm{FeH}$ is much stronger affected by this, being depleted already two pressure scale heights deeper than $\mathrm{CrH}$, which is equivalent to about one order of magnitude in optical depth. Thus, in the T1 dwarf the band is probably dominated by $\mathrm{CrH}$. We therefore conclude that we see the combined effects of $\mathrm{FeH}$ with fairly accurate opacity data, and $\mathrm{CrH}$ absorption that appears overestimated by a significant factor, in our spectral sequence. Future modelling efforts for improvement in this spectral region should therefore focus on better oscillator strengths for the $\mathrm{CrH}$ lines.

\subsubsection{Water}

Strong steam bands are visible at very low temperatures in the wavelength region redward of $9200 \AA$, where $\mathrm{TiO}$ and $\mathrm{CrH}$ are also important (see above). The model spectra provide a rather poor fit to the data, although the positions of lines are sometimes well reproduced in regions where no telluric absorption appears. However, the main contribution to the absorption in the $\mathrm{L}$ models 
turns out to be due to $\mathrm{TiO}$ and $\mathrm{CrH}$, which have both been shown to be overestimated. The total strength of the remaining flux matches quite well at large parts of these spectral regions.

Burgasser et al. (2003) identified a water band at $9250 \AA$ and Cushing \& Vacca (2006) identified absoption at $9200 \AA$ due to TiO. In our models, $\mathrm{FeH}$ and $\mathrm{CrH}$ are still strong opacity sources below $9280 \AA$, but we see very little contribution from $\mathrm{TiO}$ at $2000 \mathrm{~K}$. Hence both $\mathrm{TiO}$ and $\mathrm{FeH}$ may contribute to the absorption in this spectral region, which definitely requires further attention before it can be used for analysis.

\subsection{Atomic lines}

\subsubsection{Alkali lines}

The strongest absorption lines in the spectra of ultra cool stars are the massively pressure broadened alkali lines, i.e. elements with only one electron in their outer shell, which can get excited even at very low temperatures. All alkali elements have strong lines in the observed wavelength region. The first alkali element, $\mathrm{Li}$, has a prominent resonance line at $6708 \AA$. This line is used as an indicator of brown dwarf status in young objects (e.g. Basri et al. 2000), since evolutionary models agree that stars and brown dwarfs of more than $0.055-0.06 M_{\odot}$ will burn a significant fraction of their primordial lithium content within at most a few $10^{8}$ years (Zapatero Osorio et al. 2005). The brown dwarfs in our sample, being field objects, can be generally expected to be old and massive enough to have been depleted of most Li. This is not included in our model atmospheres, which have been calculated independently of any assumptions on the evolutionary status. The theoretical spectra thus show the Li I resonance line at full strength, while comparison with the observations demonstrates that the actual Li content is severely reduced. $\epsilon$ Indi Ba is a limiting case in this respect, as the preferred mass estimate of this brown dwarf, corresponding to a system age of $1.3 \mathrm{Gyr}$ (McCaughrean et al. 2004), places it somewhat below the $\mathrm{Li}$ burning threshold. A mass slightly above the limit is still in full agreement with observed properties and evolutionary models. $\epsilon$ Indi $\mathrm{Bb}$ contributes only $\sim 1 \%$ of the flux at this wavelength, hence its Li I line can not be observed in the combined spectrum.

The Na resonance lines at $5891 \AA$ and $5897 \AA$ are not covered by our spectra. The two subordinate lines at $8194 \AA$ and $8183 \AA$, however, are covered. In our setting, they appear just at the red end of the lower chip of the red arm. Both Na lines are clearly seen in the L0, the L2, and the L5 dwarf. In the T1 dwarf, they are detected as narrow absorption spikes. The models predict rather well the shape of these lines in the L0 and L2 dwarf while their strength is not very well fit. In the L5 and T1 dwarfs, the observed lines are broader and shallower than those predicted by the models.

The KI resonance lines at $7665 \AA$ and $7698 \AA$ govern the spectral shape of ultra-cool dwarf spectra in the optical wavelength regime. These lines have equivalent widths of several hundred Ångstrom in $\mathrm{T}$ dwarfs and are evolving from narrow lines in $\mathrm{K}$ and $\mathrm{M}$ dwarfs to very wide and smooth absorption troughs in late $\mathrm{L}$ and $\mathrm{T}$ dwarfs with wings extending more than $1000 \AA$ to the blue and especially to the red, as predicted by the line profile calculations described in Sect. 3. The qualitative behaviour of the K I doublet is reproduced well by the model fits, especially the strong pressure broadening wings in the late $\mathrm{L}$ and T dwarfs. Our new models predict the position of the $\mathrm{K}-\mathrm{H}_{2}$ quasi-molecular satellites at $6900-7100 \AA$ (at the position of the $\mathrm{O}_{2} B$-band). This wide and shallow feature, covering several echelle orders, may be present in our high-resolution spectra of $\epsilon$ Indi $\mathrm{B}$, but a detailed analysis of this feature goes beyond the scope of this paper.

The shape of the $\mathrm{K}$ lines in the early and mid-L spectra is not that well matched by the models. In the L0 dwarf, the cores of the observed $\mathrm{K}$ lines are still visible as relatively narrow absorption minima embedded in wings extending a few tens to hundred $\AA$. This broader absorption component becomes saturated already the L2 dwarf spectrum, eating up the core feature. The models qualitatively predict this effect, however, they predict the intermediate wings to be much stronger than actually observed. In the low resolution plot in Fig. 1, one can see that this part of the wings is still overpredicted in the L5 spectrum, while the far wings, that affect the spectrum all the way to the $Y$ band peak, are quite well reproduced. Since the central parts of the lines form in the highest layers of the atmosphere, especially in the early L dwarfs where alkali metals are not depleted strongly in the upper regions (Johnas et al. 2007), this is a clear indication that the Settl models do not yet produce correct densities of the neutral alkali metals in the uppermost part of the atmosphere.

Absorption lines of $\mathrm{Rb}$ at $7800 \AA$ and $7947 \AA$, and of $\mathrm{Cs}$ at $8521 \AA$ and $8943 \AA$ appear in all four spectra, even in the T dwarf spectrum. They become stronger and wider towards lower temperature. Given the fact that they are embedded in pseudocontinua that are not always a good fit to the data, the behavior of all $\mathrm{Rb}$ and $\mathrm{Cs}$ lines is very well reproduced by the models. A particularly good example is the Rb I $7800 \AA$ line in the L5 dwarf. This line very accurately reproduces the data.

\subsubsection{Other lines}

Hydrogen is observed in emission in some low mass objects, it is an indicator of a hot chromosphere that is probably magnetically heated. We find $\mathrm{H} \alpha$ in emission in our L0 and L2 spectra, which is occasionally seen in early type L dwarfs (Schmidt et al. 2007). In the two cooler objects, no line is detected. This feature does not come from the photosphere itself, hence it is not contained in the model spectra.

The next set of lines of the periodic table that can be expected in spectra of ultra cool dwarfs are the features of neutral earth alkali elements; these are $\mathrm{Be}, \mathrm{Mg}, \mathrm{Ca}, \mathrm{Sr}$, and $\mathrm{Ba} . \mathrm{Be}, \mathrm{Mg}$, and Sr do not have any transitions from low level energy states in the optical wavelength region, and we are left with transitions of $\mathrm{Ca}$ I and $\mathrm{Ba}$ I. One $\mathrm{Ca}$ I line is located at $6572 \AA$, immediately next to $\mathrm{H} \alpha$. The line is predicted in the models, it is only a little weaker than predicted in the data. Two lines of Ba I were seen by Reiners \& Basri (2006b) in an L sub-dwarf at $7912 \AA$ and $8560 \AA$. We do not see these lines in any of our spectra, which can be explained by the stronger absorption due to the metal oxides in that region against which the weak Ba lines cannot be detected (Reiners \& Basri 2006b).

Apart from these lines, we found only a set of low energy TiI lines. They are located between 9600 and $9700 \AA$ and belong to low energy transition that appears to be visible in such cool atmospheres (see also Reiners \& Basri 2006b).

The models predict a rather strong $\lambda 6894.5 \AA$ Sr I line, which we do not detect. This might indicate that the condensation chemistry of this earth alkali metal is also not fully understood, but the SNR of the observations in this region is low and telluric absorption by the $\mathrm{O}_{2} \mathrm{~B}$ band hampers a detailed comparison. 


\section{Summary}

We have presented high resolution spectra of four ultra-cool dwarfs of spectral type L and T. The complex chemistry and its feedback to the structure of such cold atmospheres is a great challenge for theorists from different areas. Our comparison to the most recent calculations showed that the general features are understood and that probably most of the species and their spectral effects are taken into account. However, in individual features, a good match between data and model spectra are still not the rule. While calculations of molecular $\mathrm{VO}$ and $\mathrm{CaH}$, and of atomic $\mathrm{Cs}$ and $\mathrm{Rb}$ are already quite satisfying, features due to molecular $\mathrm{TiO}, \mathrm{CrH}$, water, and atomic $\mathrm{Li}, \mathrm{Na}$, and $\mathrm{K}$ show large differences to the observed data.

Particularly in ultra-cool atmospheres, it is necessary to achieve a very good fit in all important absorbers in order to determine atmospheric properties, because the chemical complexity of these atmospheres reacts very sensitively on changes in any of its constituents. Thus, the full information of spectroscopic features in ultra-cool dwarfs still cannot be utilized to derive physical parameters like for example gravity, but we have clearly witnessed huge progress during the last years. With high quality spectra like the ones presented in this work, we can continue to improve detailed models of ultra-cool dwarf atmospheres.

Acknowledgements. We thank the referee, Sandy Leggett, for a very helpful report. A.R. has received research funding from the European Commission's Sixth Framework Programme as an Outgoing International Fellow (MOIF-CT2004-002544), and from the DFG as an Emmy Noether Fellow (RE 1664/4-1). We thank the Gesellschaft für Wissenschaftliche Datenverarbeitung Göttingen for generous allocation of computing time used for our calculations. This work has benefitted from the $\mathrm{M}, \mathrm{L}$, and $\mathrm{T}$ dwarf compendium housed at DwarfArchives.org. We thank Michael Cushing for pointing out the possible contribution of TiO to the absorption around $9200 \AA$.

\section{References}

Allard, F., \& Hauschildt, P. H. 1995, ApJ, 445, 433

Allard, F., Hauschildt, P. H., \& Schwenke, D. 2000, ApJ, 540, 1005

Allard, F., Hauschildt, P. H., Alexander, D. R., Tamanai, A., \& Schweitzer, A. 2001, ApJ, 556, 357

Allard, F., Guillot, T., Ludwig, H., et al. 2003, in Brown Dwarfs, ed. E. Martín, (San Francisco: Astronomical Society of the Pacific), IAU Symp., 211, 325 Allard, N. F., \& Spiegelman, F. 2006, A\&A, 452, 351

Allard, N. F., Allard, F., Hauschildt, P. H., Kielkopf, J. F., \& Machin, L. 2003, A\&A, 411, L473
Allard, N. F., Allard, F., \& Kielkopf, J. F. 2005, A\&A, 440, 1195

Allard, N. F., Allard, F., Johnas, C., \& Kielkopf, J. 2007a, A\&A, submitted

Allard, N. F., Spiegelman, F., \& Kielkopf, J. F. 2007b, A\&A, 465, 1085

Andretta, V., Gomez, M. T., \& Severino, G. 1991, Sol. Phys., 131, 1

Baraffe, I., Chabrier, G., Allard, F., \& Hauschildt, P. H. 2002, A\&A, 382, 563

Barber, R. J., Tennyson, J., Harris, G. J., \& Tolchenov, R. N. 2006, MNRAS, 368,1087

Barklem, P. S., \& O’Mara, B. J. 2001, J. Phys. B Atom. Mol. Phys., 34, 4785

Basri, G. 2000, ARA\&A, 38, 485

Basri, G., Mohanty, S., Allard, F., et al. 2000, ApJ, 538, 363

Burgasser, A. J. 2007, ApJ, 658, 617

Burgasser, A. J., Kirkpatrick, J. D., Liebert, J., \& Burrows, A. 2003, ApJ, 594, 510

Burgasser, A. J., Burrows, A., \& Kirkpatrick, J. D. 2006, ApJ, 639, 1095

Burrows, A., Sudarsky, D., \& Hubeny, I. 2006, ApJ, 640, 1063

Chabrier, G., \& Baraffe, I. 2000, ARA\&A, 38, 337

Chabrier, G., Baraffe, I., Allard, F., \& Hauschildt, P. 2000, ApJ, 542, 464

Chabrier, G., Baraffe, I., Allard, F., \& Hauschildt, P. H. 2005, [arXiv: astro-ph/0509798]

Cushing, M. C., \& Vacca, W. D. 2006, ApJ, 131, 1797

Cushing, M. C., Rayner, J. T., \& Vacca, W. D. 2005, ApJ, 623, 1115

Ferguson, J. W., Alexander, D. R., Allard, F., et al. 2005, ApJ, 623, 585

Golimowski, D. A., Leggett, S. K., Marley, M. S., et al. 2004, ApJ, 127, 3516

Hauschildt, P. H., \& Baron, E. 1999, J. Comput. Appl. Math., 109, 41

Johnas, C. M. S., Hauschildt, P. H., Schweitzer, A., et al. 2007, A\&A, 466, 323

Kirkpatrick, J. D. 2005, ARA\&A, 43, 195

Kirkpatrick, J. D., Henry, T. J., \& McCarthy, Jr., D. W. 1991, ApJS, 77, 417

Kučinskas, A., Hauschildt, P. H., Ludwig, H.-G., et al. 2005, A\&A, 442, 281

Kučinskas, A., Hauschildt, P. H., Brott, I., et al. 2006, A\&A, 452, 1021

Leininger, T., Gadéa, F., \& Dickinson, A. 2000, J. Phys. B Atom. Mol. Phys., 33, 1805

Lodders, K. 2002, ApJ, 577, 974

Lodders, K., \& Fegley, Jr., B. 2006, Chemistry of Low Mass Substellar Objects [arXiv: astro-ph/0601381]

Ludwig, H.-G., Allard, F., \& Hauschildt, P. H. 2006, A\&A, 459, 599

McCaughrean, M. J., Close, L. M., Scholz, R., et al. 2004, A\&A, 413, 1029

McLean, I. S., McGovern, M. R., Burgasser, A. J., et al. 2003, ApJ, 596, 561

McLean, I. S., Prato, L., McGovern, M. R., et al. 2007, ApJ, 658, 1217

Mohanty, S., Jayawardhana, R., \& Basri, G. 2004, ApJ, 609, 885

Reiners, A. 2005, Astron. Nachr., 326, 930

Reiners, A., \& Basri, G. 2006a, ApJ, 644, 497

Reiners, A., \& Basri, G. 2006b, AJ, 131, 1806

Schmidt, S. J., Cruz, K. L., Bongiorno, B. J., Liebert, J., \& Reid, I. N. 2007, AJ, 133,2258

Schweitzer, A., Gizis, J. E., Hauschildt, P. H., Allard, F., \& Reid, I. N. 2001, ApJ, 555, 368

Schweitzer, A., Gizis, J. E., Hauschildt, P. H., et al. 2002, ApJ, 566, 435

Schwenke, D. W. 1998, Chemistry and Physics of Molecules and Grains in Space, Faraday Discuss., 109, 321

Tinney, C. G., \& Reid, I. N. 1998, MNRAS, 301, 1031

Vrba, F. J., Henden, A. A., Luginbuhl, C. B., et al. 2004, AJ, 127, 2948

Zapatero Osorio, M. R., Martín, E. L., Lane, B. F., et al. 2005, Astron. Nachr., 326,948 